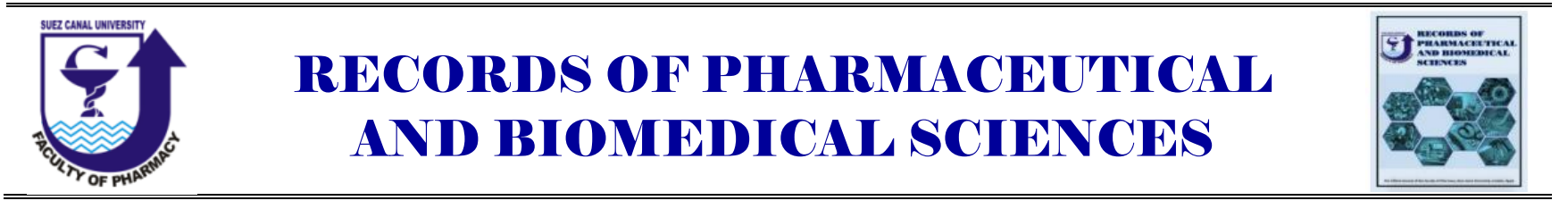

\title{
Anti-Diabetic Medications: A promising therapeutic approaches for the Management of NAFLD
}

\author{
Ahmed Z. Ibrahim ${ }^{a}$, Reem M. Hazem ${ }^{a}$, Dina A. Ali ${ }^{b}$, Yasser M. Mostafa ${ }^{a}$ \\ ${ }^{a}$ Department of Pharmacology \& Toxicology, Faculty of Pharmacy, Suez Canal University, Ismailia 41522, \\ Egypt, ${ }^{b}$ Department of Clinical Pharmacology, Faculty of Medicine, Suez Canal University, Ismailia 41522, \\ Egypt
}

Received on: 06. 06. 2021

Revised on: 15.062021

Accepted on: 20. 06. 2021

*Correspondence Author:

Tel: +2-010-92485596

E-mail address:

ahmed.z.ibrahim@pharm.suez.edu.eg

\begin{abstract}
Type 2 diabetes and Nonalcoholic fatty liver disease are two of the most common metabolic diseases in the world, with an increasing prevalence. Patients with T2DM have a two-fold increased risk of developing NAFLD, The evidence that certain antidiabetic drugs boost NAFLD/NASH in T2DM patients is growing. However, there are no specific pharmacologic treatments available. This review aims to outline the data supporting the effectiveness of anti-diabetic drugs in the treatment of NAFLD, besides providing an overview of novel antidiabetic therapies that have been proposed to achieve this goal. Most of these anti-diabetic agents demonstrate short-term efficacy, but have shown little to no impact on hepatic histology. Only thiazolides and GLP-1 receptor agonists have shown considerable improvement in hepatic histology.
\end{abstract}

Keywords: GLP-1, Steatohepatitis, Diabetes, NAFLD

\section{Introduction}

It was thought that nonalcoholic steatohepatitis (NASH) is a rare symptom that only affected women with second type of diabetes in 1990. In 1996, a study identified NASH as the second most frequently occurring liver disease in patients, behind acute and chronic viral hepatitis. NASH disorders like fibrosis and cirrhosis range from 15$50 \%$ in all patients (Byron \& Minuk, 1996).

The two-hit theory by Day and James, states that the pathogenesis of NASH begins with a first hit triggered by lipid accumulation in hepatocytes, resulting in apoptosis and enhanced oxidative stress (Marra, 2004).

Steatosis therefore predisposes the liver to produce progressive NASH because of a second attack from invading immune cells that activate proinflammatory mediators like cytokines. This transition to inflammatory state results in fibrotic tissue remodeling, that can rapidly lead to final liver disease, like cirrhosis and the progression of hepatocellular carcinoma (HCC) (Drescher et al., 2019).

Today, this theory is believed insufficient to account for the multiple disease drivers associated with NAFLD (Nonalcoholic fatty liver disease) (Buzzetti et al., 2016). Recently, the multiple-hit theory has given a more complete picture of the diverse conditions that leads to the growth and pathogenesis.

However, evidence suggests that growth of the 
disease is not always linear, and who is more likely to develop to final stages is not exactly clear. NAFLD, that is closely correlated to type 2 diabetes and dyslipidemia, is considered the hepatic form of metabolic syndrome (Drescher et al., 2019).

Obesity, insulin resistance, and dyslipidemia are the most well-known risk factors for NAFLD (Lin et al., 2011). Moreover, genetic modifiers are linked to an increased risk of NASH and cirrhosis. The liver's lipid resource is influenced by dietary lipids (15\%), lipoid tissue lipolysis (60-80\%), and de novo lipogenesis $(5 \%)$. Adipose tissue lipolysis is regulated by insulin's effects on adipocytes regulate (Smith et al., 2020).

The hepatic receptor (intervening in liver fatty acid production), the farnesoid receptor (that interfere in the formation of VLDL), and the peroxisome proliferator-activated receptors [PPARs] are all atomic receptors and cytoplasmic transcription parameters that regulate de novo lipogenesis (Sanders \& Griffin, 2016).

PPAR- regulates the oxidation of free fatty acids, acts as an anti-inflammatory factor, inhibits hepatocellular lipogenesis and suppresses proinflammatory expression of genes in the liver (Kawaguchi \& Torimura, 2020).

Steatosis is the accumulation of lipid in liver cells with an abnormally high level of triglycerides as a result of an abnormal lipid metabolism. Steatosis is caused by insulin resistance. Insulin resistance promotes hepatic triglyceride accumulation by raising peripheral adipose tissue lipolysis, contributing to the rise of circulating non-esterified fatty acids that the liver excretes and converts to triglycerides. When insulin signaling is impaired, compensatory hyperinsulinemia happens (Giorgio et al., 2013).

Hyperinsulinemia decreases glycogen synthesis, which increases hepatic fatty acid intake, alters triglyceride transfer, and decreases hepatic oxidation. Additionally, glucose can be absorbed by the liver by an insulin-independent receptor and converted to pyruvate (a precursor of acetyl-CoA and malonyl-CoA), that can be converted to fatty acids by de novo lipogenesis (Fernando et al., 2019).

The first step in NAFLD pathogenesis is rise in pro-inflammatory cytokines which results in oxidative stress-induced lipotoxicity, impaired hepatocyte apoptosis, activation, and mitochondrial dysfunction. This leads to fatty acid deposition, hepatocellular disruption, proliferation, and persistent extracellular. The second step is oxidative stress, which is induced by proinflammatory cytokines. As a consequence, insulin resistance and liver cell damage induced by environmental or genetic preferences can be intensified (Duvnjak, 2007).

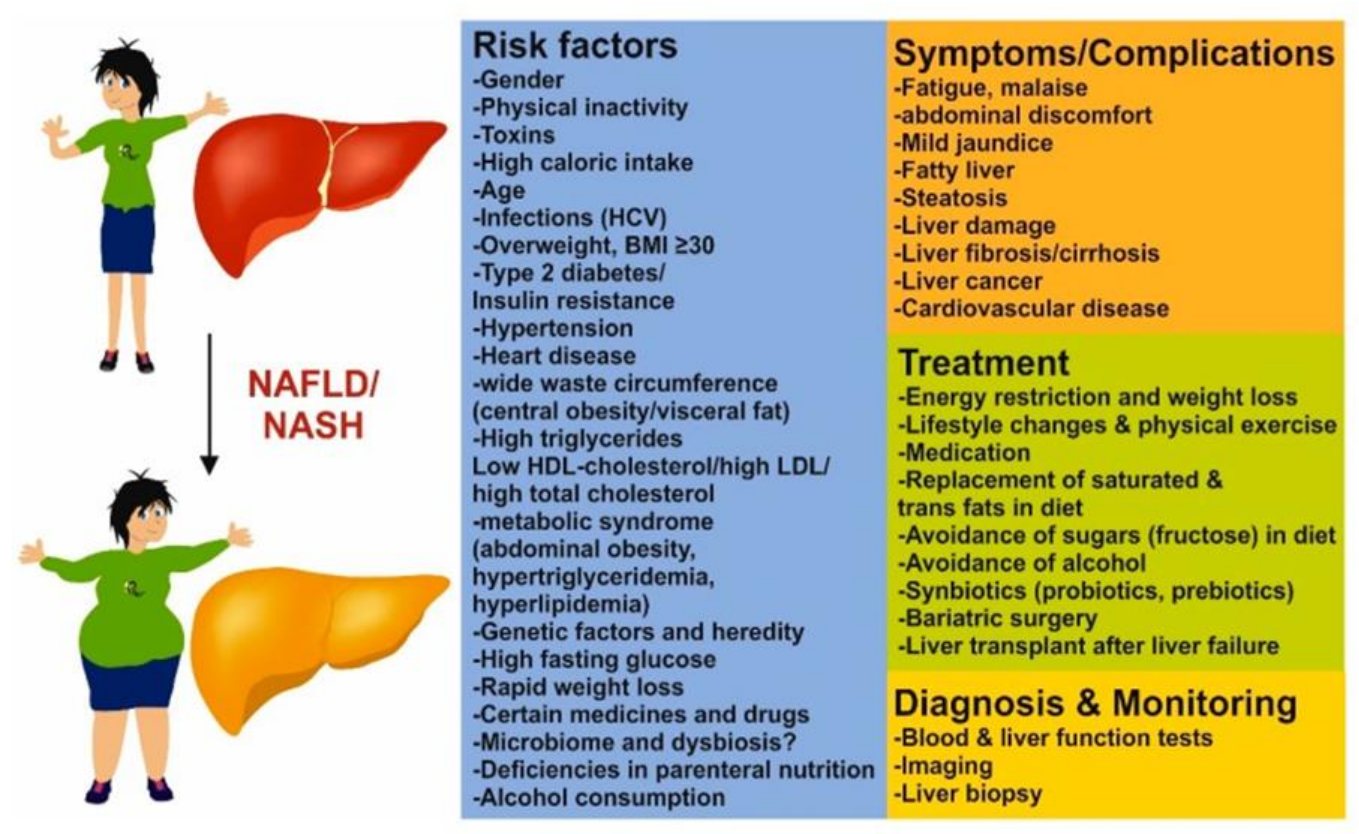

Figure 1: Risk factors, symptoms, diagnosis, and treatment of Nonalcoholic liver symptoms (Allard et al., 2019) 
Rec. Pharm. Biomed. Sci. 5 (3), 65-70, 2021

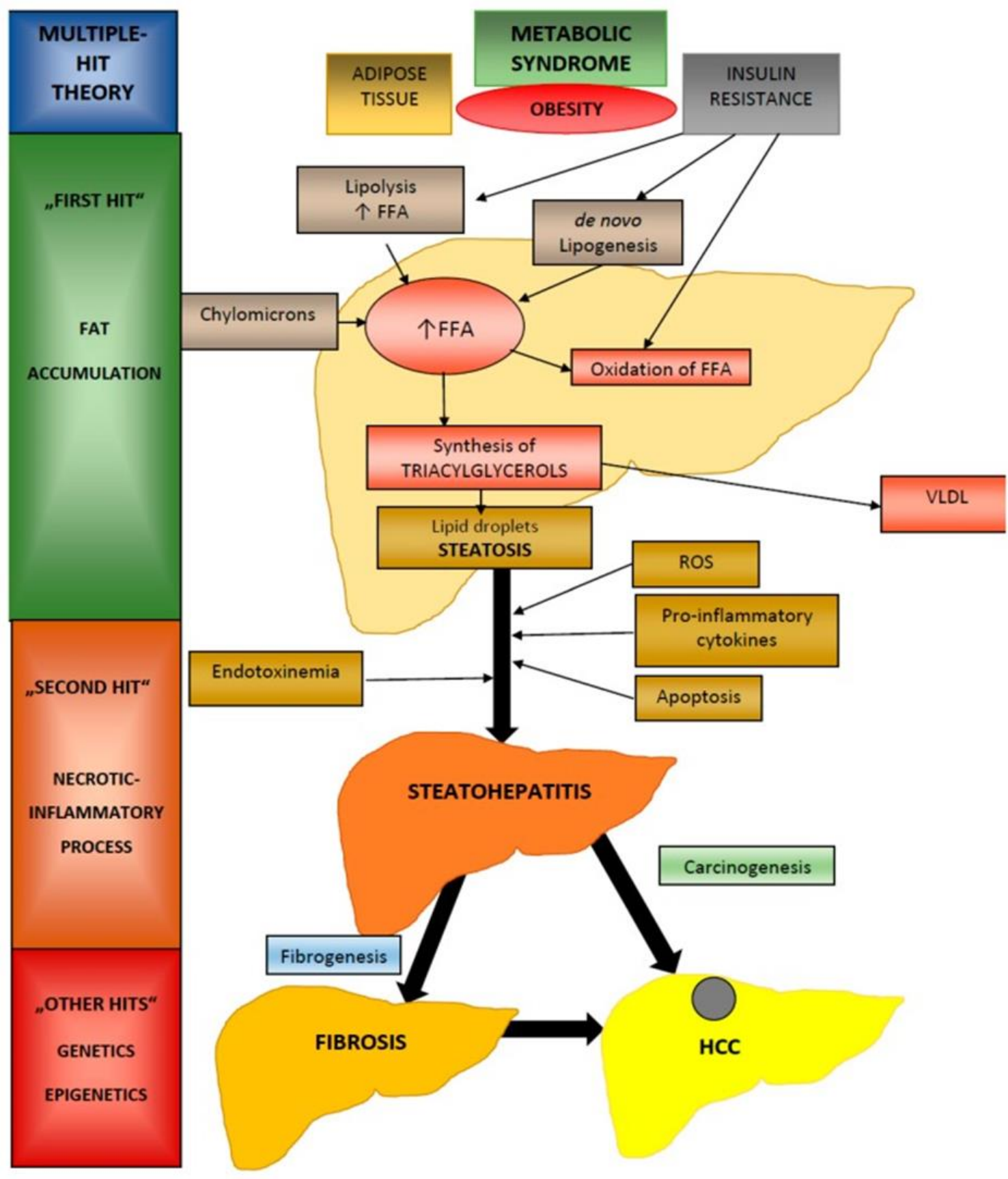

Figure 2: The pathogenesis of NAFLD (Marchisello et al., 2019)

\section{Treatment of NAFLD}

There is no single remedy for NAFLD that has been shown to be completely successful. The main therapeutic objective is to improve steatosis and avoid the disease progression. Intensive lifestyle change and treatment of the risk factors are the basics of disease management. Medical and surgical procedures serve as second-line treatments, or as adjuvants (Pappachan et al., 2017).

\subsection{Metformin}

Metformin treatment was linked to insulin resistance and liver transaminases changes, but it did not alter histological factors including steatosis, inflammation, hepatocellular ballooning, and fibrosis. Metformin is recommended for T2DM patients or even prediabetes conditions and NAFLD for its antidiabetic potency. Metformin has been shown to be effective, including in cirrhosis 
patients, and may prevent T2DM patients and longterm hepatic diseases from developing HCC (Bhat, 2015).

\subsection{Thiazolidinediones}

These medications enhance blood glucose regulation by modulating tissue insulin tolerance through the (PPAR)- $\gamma$ signaling pathway. Rosiglitazone and pioglitazone have been extensively studied in this section of medication for T2DM therapy (Greenfield \& Chisholm, 2004).

Controversy over elevated cardiac disorders, rosiglitazone usage is recently dropped rapidly, with pioglitazone now becoming the most prescribed compound. Liver insulin resistance and fatty acid oxidation have been improved, and liver lipogenesis has been inhibited, according to pioglitazone (Norris et al., 2007).

\subsection{Incretin-based therapy}

The GLP-1 analogues like "exenatide, liraglutide, dulaglutide and semaglutide" and dipeptidyl peptidase-4 (DPP-4) inhibitors like "sitagliptin, saxagliptin, vildagliptin, alogliptin and linagliptin" are two major classes of incretin-related medications that have been thoroughly examined for use in NAFLD (Carbone et al., 2016).

These medications increase pancreatic insulin secretion in response to meals, as well as having extra-pancreatic impacts on several organs, making them extremely effective in the treatment of T2DM. GLP-1 analogues are linked to fat burning, whereas DPP-4 inhibitors have no effect on weight. Many overweight/obese T2DM patients have NAFLD and are treated with incretin-based therapy (Blaslov, 2014).

\subsection{Sodium glucose cotransporter 2 inhibitors}

Dapagliflozin (Forxiga) is just one of a new category of glucose-lowering drugs called sodiumglucose co-transporter-2 (SGLT2) inhibitors, which is used to treat type 2 diabetes patients. Dapagliflozin inhibits the transporter protein SGLT2 in the kidneys, leading to higher urinary glucose excretion and decreased blood glucose rates (Filippatos et al., 2015).

Dapagliflozin's efficiency is not affected by insulin discharge and activity. As a result, dapagliflozin offers effective treatment when it is used in conjunction with other antidiabetic medications due to its particular mode of action (Papakitsou et al., 2019). Dapagliflozin has also been shown to improve insulin sensitivity (Merovci et al., 2016).

The SGLT2 inhibitors not only reduce body overweight but also reduce liver fat accumulation in NAFLD and T2DM patients. The majority of losing weight was due to a reduction in fat mass rather than skeletal muscle mass (Choi et al., 2018).

\section{Conclusion}

Anti-diabetic medications are considered as a successful choice for the treatment of NAFLD. Since T2DM and NAFLD have a partially shared pathophysiology that involves insulin tolerance, The majority of anti-diabetic medications improved liver enzymes, but there is no evidence that they have any impact on histological outcomes. Dapagliflozin has shown improvement in liver histology in patients with biopsy-proven NASH, making it the best anti-diabetic agent for treating NAFLD.

\section{References:}

Allard, J., Le Guillou, D., Begriche, K., \& Fromenty, B. (2019). Drug-induced liver injury in obesity and nonalcoholic fatty liver disease. In Advances in Pharmacology (Vol. 85, pp. 75-107). Elsevier.

https://doi.org/10.1016/bs.apha.2019.01.003

Bhat, A. (2015). Systematic review: Preventive and therapeutic applications of metformin in liver disease. World Journal of Hepatology, 7(12), 1652. https://doi.org/10.4254/wjh.v7.i12.1652

Blaslov, K. (2014). Incretin based therapies: A novel treatment approach for non-alcoholic fatty liver disease. World Journal of Gastroenterology, 20(23), 7356.

https://doi.org/10.3748/wjg.v20.i23.7356

Buzzetti, E., Pinzani, M., \& Tsochatzis, E. A. (2016). The multiple-hit pathogenesis of nonalcoholic fatty liver disease (NAFLD). Metabolism: Clinical and Experimental, 65(8), 1038-1048.

https://doi.org/10.1016/j.metabol.2015.12.012 
Byron, D., \& Minuk, G. Y. (1996). Clinical hepatology: Profile of an urban, hospital-based practic. Hepatology, 24(4), 813-815. https://doi.org/10.1002/hep.510240410

Carbone, L. J., Angus, P. W., \& Yeomans, N. D. (2016). Incretin-based therapies for the treatment of non-alcoholic fatty liver disease: A systematic review and meta-analysis: Incretin therapies in fatty liver disease. Journal of Gastroenterology and Hepatology, 31(1), 23-31. https://doi.org/10.1111/igh.13026

Choi, D.-H., Jung, C.-H., Mok, J.-O., Kim, C.-H., Kang, S.-K., \& Kim, B.-Y. (2018). Effect of Dapagliflozin on Alanine Aminotransferase Improvement in Type 2 Diabetes Mellitus with Non-alcoholic Fatty Liver Disease. Endocrinology and Metabolism, 33(3), 387. https://doi.org/10.3803/EnM.2018.33.3.387

Drescher, H., Weiskirchen, S., \& Weiskirchen, R. (2019). Current Status in Testing for Nonalcoholic Fatty Liver Disease (NAFLD) and Nonalcoholic Steatohepatitis (NASH). Cells, 8(8), 845. https://doi.org/10.3390/cells8080845

Duvnjak, M. (2007). Pathogenesis and management issues for non-alcoholic fatty liver disease. World Journal of Gastroenterology, 13(34), 4539. https://doi.org/10.3748/wjg.v13.i34.4539

Fernando, D. H., Forbes, J. M., Angus, P. W., \& Herath, C. B. (2019). Development and Progression of Non-Alcoholic Fatty Liver Disease: The Role of Advanced Glycation End Products. International Journal of Molecular Sciences, 20(20), 5037. https://doi.org/10.3390/ijms20205037

Filippatos, T. D., Liberopoulos, E. N., \& Elisaf, M. S. (2015). Dapagliflozin in patients with type 2 diabetes mellitus. Therapeutic Advances in Endocrinology and Metabolism, 6(1), 29-41. https://doi.org/10.1177/2042018814558243

Giorgio, V., Prono, F., Graziano, F., \& Nobili, V. (2013). Pediatric non alcoholic fatty liver disease: Old and new concepts on development, progression, metabolic insight and potential treatment targets. BMC Pediatrics, 13(1), 40. https://doi.org/10.1186/1471-2431-13-40
Greenfield, J. R., \& Chisholm, D. J. (2004). Experimental and clinical pharmacology: Thiazolidinediones - mechanisms of action. Australian Prescriber, 27(3), 67-70. https://doi.org/10.18773/austprescr.2004.059

Kawaguchi, T., \& Torimura, T. (2020). Is metabolic syndrome responsible for the progression from NAFLD to NASH in non-obese patients? Journal of Gastroenterology, 55(3), 363-364. https://doi.org/10.1007/s00535-019-01650-1

Lin, Y.-C., Chou, S.-C., Huang, P.-T., \& Chiou, H.-Y. (2011). Risk factors and predictors of nonalcoholic fatty liver disease in Taiwan. Annals of Hepatology, 10(2), 125-132. https://doi.org/10.1016/S1665-2681(19)31560-1

Marchisello, S., Pino, A. D., Scicali, R., Urbano, F., Piro, S., Purrello, F., \& Rabuazzo, A. M. (2019). Pathophysiological, Molecular and Therapeutic Issues of Nonalcoholic Fatty Liver Disease: An Overview. International Journal of Molecular Sciences, 20(8), 1948. https://doi.org/10.3390/ijms20081948

Marra, F. (2004). NASH: Are genes blowing the hits? Journal of Hepatology, 40(5), 853-856. https://doi.org/10.1016/j.jhep.2004.03.005

Merovci, A., Abdul-Ghani, M., Mari, A., SolisHerrera, C., Xiong, J., Daniele, G., Tripathy, D., \& DeFronzo, R. A. (2016). Effect of Dapagliflozin With and Without Acipimox on Insulin Sensitivity and Insulin Secretion in T2DM Males. The Journal of Clinical Endocrinology \& Metabolism, 101(3), 1249-1256. https://doi.org/10.1210/jc.2015-2597

Norris, S., Carson, S., \& Roberts, C. (2007). Comparative Effectiveness of Pioglitazone and Rosiglitazone in Type 2 Diabetes, Prediabetes,and the Metabolic Syndrome: A Meta-Analysis. Current Diabetes Reviews, 3(2), 127-140. https://doi.org/10.2174/157339907780598216

Pappachan, J. M., Babu, S., Krishnan, B., \& Ravindran, N. C. (2017). Non-alcoholic Fatty Liver Disease: A Clinical Update. Journal of Clinical and Translational Hepatology, 5(4), 384-393. https://doi.org/10.14218/JCTH.2017.00013 
Sanders, F. W. B., \& Griffin, J. L. (2016). De novo lipogenesis in the liver in health and disease: More than just a shunting yard for glucose. Biological Reviews, 91(2), 452-468. https://doi.org/10.1111/brv.12178

Smith, G. I., Shankaran, M., Yoshino, M., Schweitzer, G. G., Chondronikola, M., Beals, J. W., Okunade, A. L., Patterson, B. W., Nyangau, E., Field, T., Sirlin, C. B., Talukdar, S., Hellerstein, M. K., \& Klein, S. (2020). Insulin resistance drives hepatic de novo lipogenesis in nonalcoholic fatty liver disease. Journal of Clinical Investigation, 130(3), 1453-1460.

https://doi.org/10.1172/JCl134165 